\title{
Respiratory component of the orienting reflex: a novel sensitive index of sensory-induced arousal in rats
}

\section{Eugene Nalivaiko ${ }^{1}$ *, Evgeny Bondarenko ${ }^{1}$, Andreas Lidström ${ }^{1}$ and Robert J. Barry ${ }^{2}$}

School of Biomedical Sciences and Pharmacy, University of Newcastle, Newcastle, NSW, Australia

${ }^{2}$ Brain \& Behaviour Research Institute, School of Psychology, University of Wollongong, Wollongong, NSW, Australia

\section{Edited by:}

Elisabeth Lambert, Baker IDI Heart and Diabetes Institute, Australia

\section{Reviewed by:}

Fernando Morgan De Aguiar Corrêa, University of Sao Paulo, Brazil

Thomas E. Dick, Case Western

Reserve University, USA

${ }^{*}$ Correspondence:

Eugene Nalivaiko, School of

Biomedical Sciences, Flinders

Medical Centre, University of

Newcastle, Callaghan, NSW 2308,

Australia.

e-mail:eugene.nalivaiko@

flinders.edu.au
In humans, the integrated response to a novel stimulus (orienting reflex, OR) includes behavioral (head turning etc.) and well-characterized physiological components (changes in heart rate, respiration, skin conductance, and EEG patterns). In rodents, the physiological components of the OR include changes in heart rate and cutaneous vasoconstrictor tone, but respiratory changes have so far not been systematically documented. In the present study conducted in adult male Wistar rats, the OR was elicited by $60-\mathrm{dB}$ acoustic tones while animals were in a whole-body plethysmograph for respiratory recordings. In addition to respiration, in different groups of animals we concurrently recorded either EEG, or heart rate (both by biotelemetry), or tail blood flow (using ultrasound Doppler). Acoustic stimuli provoked vigorous tachypneic responses with respiratory rate rising from 80-100 to $450-650 \mathrm{cpm}$, and with small and variable changes in tidal volume. This respiratory arousal response was often, but not always, accompanied by EEG desynchronization and by variable tail vasoconstriction, and by small and inconsistent changes in the heart rate. We conclude that tachypneic responses are a new highly sensitive index of sensory-induced arousal.

Keywords: arousal, orienting response, respiratory rate, sniffing, EEG, ECG, tail blood flow

\section{INTRODUCTION}

The orienting reflex (OR), originally described by Pavlov in 1910 as the "What is that?" reflex (see Pavlov, 1927), is of continued interest in modern psychophysiology and cognitive neuroscience. Although initially conceptualized in behavioral terms (turning or orienting toward a novel stimulus), its physiological correlates were emphasized by the late Russian researcher, Evgeni N. Sokolov. Sokolov $(1960,1963 \mathrm{a}, \mathrm{b})$ identified the OR as the major unit of perceptual functioning, reflexively directing the organism's attention to important events in the environment. Sokolov considered the OR as a whole-of-body reflex, involving particular changes in a large range of physiological systems. One of Sokolov's important generalizations was that this complex phenomenon was considered a unitary system, and always referred to in the singular sense.

Systematic testing revealed inconsistencies in Sokolov's theory. For example, Barry (1977a) presented each of 24 subjects with one of the $4 !(4 \times 3 \times 2 \times 1=24)$ unique cycles of $20,30,40$, and $50 \mathrm{~dB}$ auditory tones $(1000 \mathrm{~Hz})$ at long variable inter-stimulus intervals. Each subject received 8 repetitions of their cycle in a continuous sequence to explore response decrement. The electrodermal skin conductance response, respiratory pause, peripheral vasoconstriction in the fingers, cephalic vasodilation in the temporal area, EEG alpha desynchronization, and heart rate deceleration were examined at each stimulus presentation. The first stimulus produced brief phasic responses in all these systems. But beyond this initial response, differences in stimulus parameters produced response patterns differing between the measures. Indeed, across the six measures, four patterns over the two independent variables were found: (i) no change with intensity or trial (heart rate deceleration, cephalic vasodilation); (ii) no change with intensity, but reduction over trials (respiratory pause, alpha desynchronization); (iii) sensitive to intensity, but not trial repetition (peripheral vasoconstriction); (iv) sensitive to intensity and trial repetition (skin conductance response). This pattern of response fractionation was confirmed in subsequent studies (Barry, 1977b, 1978, 1979; Barry and James, 1981). This fourfold pattern of response fractionation, opposing Sokolov's unitary OR generalization, was found to be reliable, and led to the formulation of alternative accounts of stimulus-response patterning in the OR context, eventually developing into what is now known as Preliminary Process Theory (see Barry, 2006, 2009). The essence of that work is in conceptualizing experimental observations that different components of the OR reflect different dimensions of corresponding sensory stimuli. For example, cardiac deceleration reflects detection of a stimulus; respiratory and EEG components - novelty, and cutaneous vasoconstriction - stimulus intensity. This theory has serious implications and consequences for experimental work, as it postulates, in contrast to Sokolov's ideas, that different brain mechanisms (and possibly different brain areas) are engaged in the generation of different components of the OR.

The arousal-related response fractionation that forms the basis of the Preliminary Process Theory has never been assessed in animal studies, and, surprisingly, an integrative approach to studies of the OR is rarely used in animal experiments. Indeed, while individual components - usually either HR or EEG - have been examined 
in great detail, their inter-relationship, and relations to other OR components, remains unknown. In a recent study conducted in rats we found dissociation between respiratory and cardiac OR components in rats (Kabir et al., 2010), an observation supporting Barry's fractionation and opposing Sokolov's unitary concept. In order to further validate the response-fractionation basis of the PPT theory, in this study we aimed to complement these previous finding with a broader range of arousal-related physiological measures. To this end, we recorded EEG, respiration, heart rate, and cutaneous vasoconstriction responses to readily quantifiable mild acoustic stimuli in conscious rats.

\section{MATERIALS AND METHODS ANIMALS AND PRELIMINARY SURGERY}

Male Sprague Dawley rats weighing 280-320 g were used in all experiments. All efforts were made to reduce animal pain or discomfort. The experimental protocol was approved by the University of Newcastle (Experiment 2) or Flinders University (Experiments 1 and 3) Animal Ethics Committees, and is in compliance with the European Communities Council Directive of 24 November 1986 (86/609/EEC). Preparatory surgery was conducted under isoflurane (1.5\% in $100 \%$ oxygen) anesthesia, with carprofen $(5 \mathrm{mg} / \mathrm{kg}$ ) being used as a post-surgery analgesic. In the first group of rats (EEG study), telemetric radio-transmitters for recording biopotentials (TA11CA-F40, Data Sciences International, DSI) were implanted in the peritoneal cavity, with leads tunneled subcutaneously to the top of the skull. The electrodes were placed epidurally according to DSI recommendations (one $2.0 \mathrm{~mm}$ anterior to Bregma and $1.5 \mathrm{~mm}$ lateral, another $7.0 \mathrm{~mm}$ posterior to Bregma and $1.5 \mathrm{~mm}$ lateral) and fixed to the skull with dental cement and screws. In the second group of rats (ECG study) similar telemetric transmitters we used, with electrodes positioned in accord with the method described by Sgoifo et al. (1996): one electrode on the internal surface of the xiphoid process, another in the mediastinum, along the trachea at the level of the left ventricle. This placement allows reliable recordings even in vigorously moving animals. In the third group of rats (tail blood flow study), a 1-mm i.d. ultrasonic Doppler flow probe (Iowa Doppler Products, IA, USA) was implanted around the tail artery about $3 \mathrm{~cm}$ from the base (Garcia et al., 2001); the leads were tunneled under the skin and exteriorized at the back of the neck. Upon recovery from anesthesia, animals were returned to the animal house for at least 1 week before experiments began.

\section{RECORDINGS OF RESPIRATION AND GROSS MOTOR ACTIVITY}

Respiratory movements were detected using a custom-built wholebody plethysmograph (Kabir et al., 2010). This consisted of a sealed Perspex cylinder (i.d. $95 \mathrm{~mm}$, length $260 \mathrm{~mm}$, volume $2.5 \mathrm{l}$ ) with medical air constantly flushed through it at a flow rate of $2.5 \mathrm{l} / \mathrm{min}$. A plastic T-connector was inserted $20 \mathrm{~cm}$ from the start of the output line and then linked to one input of a differential pressure amplifier (model 24PC01SMT, Honeywell Sensing and Control, Golden Valley, MN, USA), the second input being open to the room air. For semi-quantitative assessment of animals' motor activity, a piezoelectric pulse transducer (MLT1010/D, ADInstruments, Sydney, Australia) was placed under the plethysmograph. The transducer was sensitive enough to detect even minor movements (e.g., turning the head), while locomotion produced large oscillatory responses.

\section{EXPERIMENTAL PROTOCOL, DATA ACQUISITION, AND ANALYSIS}

After turning on telemetric transmitters for EEG (Experiment 1) or ECG (Experiment 2) recordings, rats were placed into the plethysmographic chamber that in turn was positioned on the radiotelemetric receiver. In Experiment 3, prior to placement in the chamber, exteriorized leads from the Doppler probe were connected to a pulsed Doppler flowmeter (Model 202, Triton technology, Inc., CA, USA). In all experiments, basal recordings were conducted for $25-30 \mathrm{~min}$, and then acoustic stimuli (60 or $90 \mathrm{~dB}$ white noise, $0.5 \mathrm{~s}$ duration with $50 \mathrm{~ms}$ rise and fall times) were presented at random intervals, $4-5 \mathrm{~min}$ apart. A $60-\mathrm{dB}$ sound intensity is well above threshold but does not provoke the startle response. The stimuli were presented when animals were in a quiet wake state - i.e., no motor activity, eyes opened, slow regular breathing.

Analog respiratory, EEG, ECG, tail blood flow, and motion signals were digitized at $1 \mathrm{KHz}$ and acquired using a PowerLab A/D converter and Chart Pro 6.0 software (ADInstruments, Sydney, Australia). Computations of respiratory rate, EEG spectral power and of heart rate were performed using the same software.

One-way ANOVAs followed by post hoc Bonferroni's comparison test were used for assessing significance of changes in physiological variables. Data values are presented as Mean \pm SEM. Differences were considered significant at $p<0.05$.

\section{RESULTS}

Mean values for respiratory rate and heart rate were $95 \pm 8 \mathrm{cpm}$ and $346 \pm 11 \mathrm{bpm}$, respectively. Similar to our previous reports (e.g., Garcia et al., 2001; Ootsuka et al., 2008), the tail blood flow signal was highly variable in each animal, with periods of vasodilation (45-60 kHz shift) intermingled with periods of vasoconstriction, when the pulsatile signal was barely detectable.

In all rats, the acoustic stimuli provoked well defined tachypneic responses (Figures 1-3). These responses occurred at relatively short latencies ( $97 \pm 12 \mathrm{~ms}$, range 48-196 ms) and varied in amplitude: respiratory rate increased from $80-110 \mathrm{cpm}$ at rest to a maximum of 350-600 cpm and then gradually returned to baseline within several seconds. Of note, the rise of respiratory rate was not gradual, i.e., it reached maximum within a single respiratory cycle. Tidal volume either remained unchanged or increased by 20-30\%. Responses to the $60-\mathrm{dB}$ stimulus often occurred with minimal or no body movement (Figure 1).

In experiments with concurrent recording of EEG and respiration, tachypneic respiratory responses were always associated with EEG desynchronization, with larger suppression of EEG amplitude at the lower frequency range (Figure 1). Mean reduction of the total spectral power was from $397 \pm 25$ to $181 \pm 14 \mu \mathrm{V}^{2}$.

In the second group of rats, we simultaneously recorded respiration and ECG. We did not find any consistent effects of our acoustic stimulation on the heart rate, even when respiratory responses were quite vigorous (Figure 2). There were no obvious cardiac responses immediately (first 2-3 s) after the stimulus; small tachycardia (10-15 bpm) sometimes developed a few seconds later.

Acoustic stimuli provoked variable effects in the tail blood flow, depending on the basal level of this variable. When the 

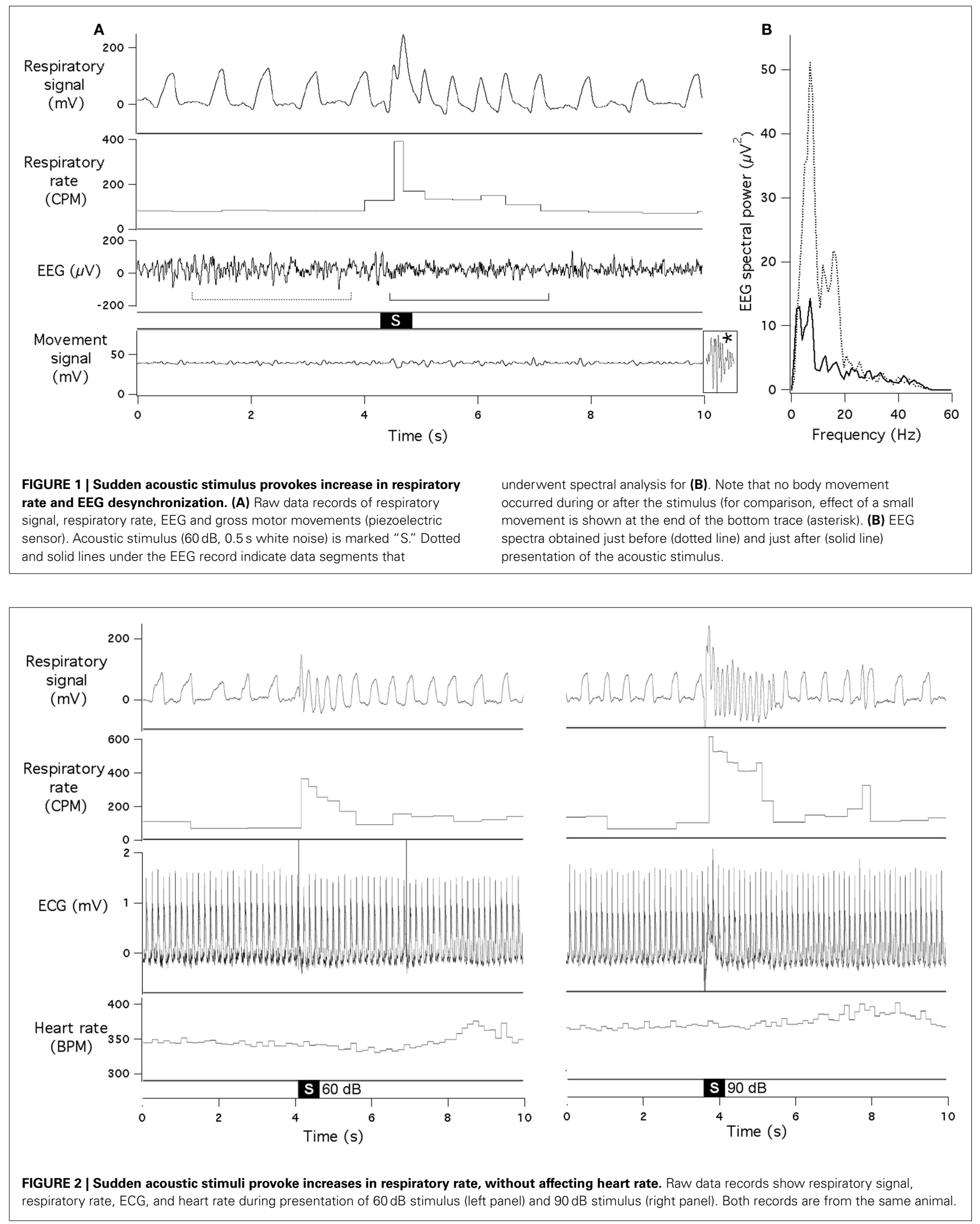

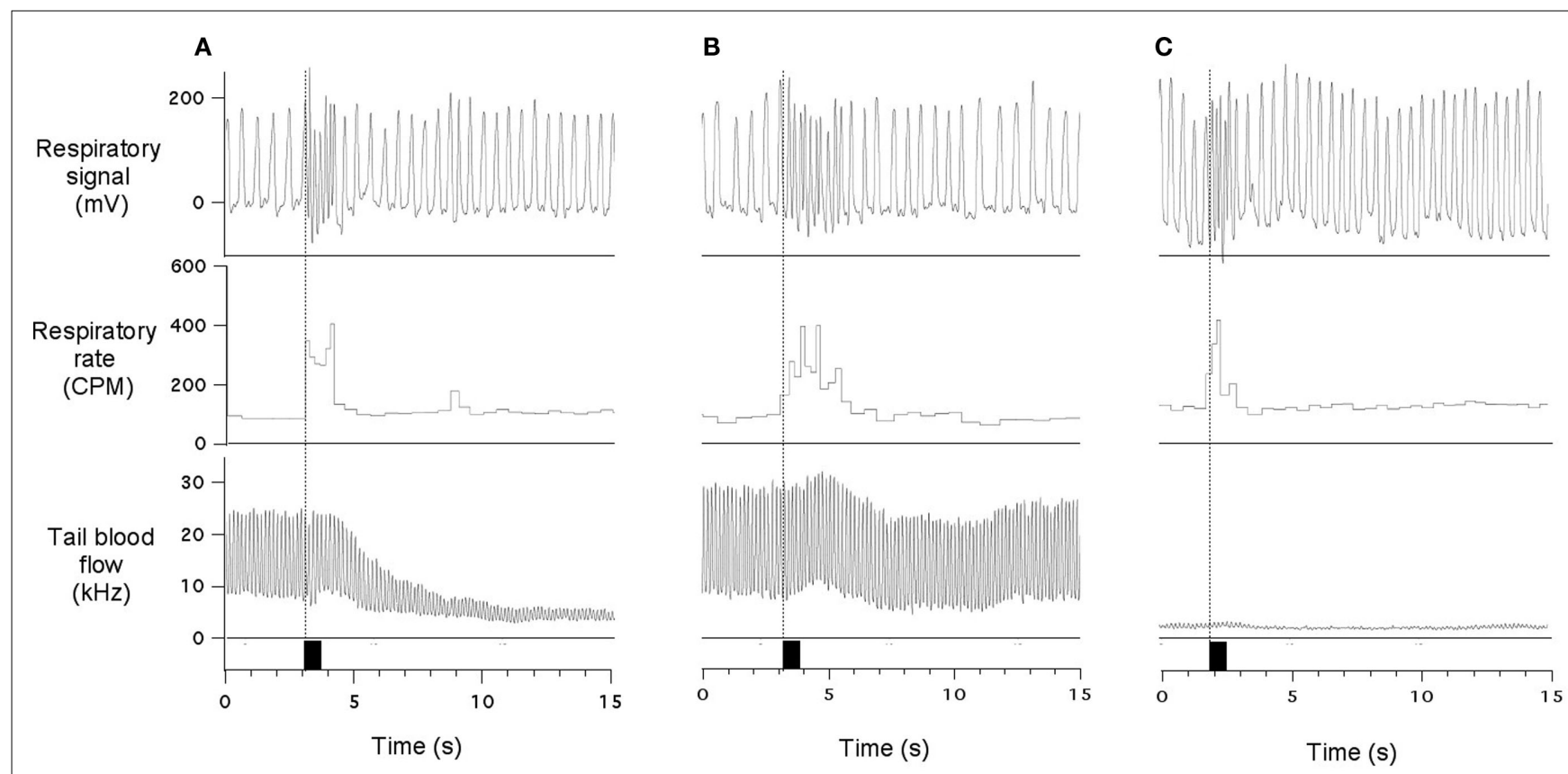

FIGURE 3 | Sudden acoustic stimuli provoke increases in respiratory rate associated with variable effects on the cutaneous blood flow. In (A), stimulus provoked vigorous vasoconstriction in the cutaneous vascular bed; in (B) - very modest cutaneous effect was observed; and in (C) - cutaneous

reaction is undetectable due to very low baseline blood flow. Data traces show respiratory signal, respiratory rate and ultrasound Doppler signal from the tail artery. Black rectangles indicate presentation of acoustic stimuli $(60 \mathrm{~dB})$. All records are from the same experiment. See text for details.

flow was at reasonably high levels, the stimuli usually provoked a substantial reduction (Figure 3A); the onset of this effect was delayed by $1.5-2 \mathrm{~s}$ compared to respiratory responses. The time course of this cutaneous vasoconstrictor response was substantially slower than the respiratory effects. Sometimes vasoconstriction was quite modest or even absent while the tachypneic response was still vigorous (Figure 3B). Finally, when the flow was at near-zero levels, acoustic stimuli had no effect on it (Figure 3C) while respiratory responses were well expressed. Thus, tachypneic respiratory responses could be associated with prominent, modest, or no cutaneous response.

\section{DISCUSSION}

Our principal purpose was to determine the relationship between individual components of the orienting response in rats. Our major novel findings are that rapid "sniffing" respiratory arousal responses to innocuous auditory stimuli are associated with cortical arousal. They are more sensitive than HR or cutaneous vasoconstriction responses, and have temporal resolution comparable to EEG desynchronization.

\section{RESPIRATORY AROUSAL IS NOT A STARTLE}

We have recently described respiratory arousal responses elicited in rats by sudden sensory stimuli of various modalities (Kabir et al., 2010), and our current data expand the range of these stimuli to the purely acoustical. Several lines of evidence suggest that this response form is not a simple startle reflex. Firstly, their latencies are considerably longer than latencies of startle response in rats (6-8 ms, Davis, 1986). Secondly, the intensity of our acoustic stimuli $(60 \mathrm{~dB})$ and their slow rise and fall times were deliberately selected to avoid startle, and indeed in many instances tachypneic responses occurred without any associated motor responses. Finally, in a preliminary study we have demonstrated that integrity of the dorsomedial hypothalamus (DMH; a well known "defense area" engaged in cardiovascular responses to stressors; Hilton, 1982; DiMicco et al., 2002) is also essential for the expression of this respiratory response form (Nalivaiko et al., 2010). In contrast, the reflex arc of the startle response is limited to the lower pons (Davis, 1986). It is thus quite likely that some simple reflexive processing of the stimulus event is involved in the genesis of these rapid respiratory responses, as in rodents, sniffing elicited by non-olfactory stimuli most probably serves a risk-assessment purpose. Of major interest here is that qualitatively similar tachypneic responses have been reported in newborn infants in response to tactile stimuli (McNamara et al., 1998), and in a rudimentary form they are present in adult humans (Harver and Kotses, 1987; Reyes del Paso and Vila, 1993).

\section{THE IMPORTANCE OF THIS NEW RESPIRATORY RESPONSE}

In contrast to psychophysiological studies in human, the means of determining sensory arousal in experimental animals are limited. The simplest and most evident relies on motor responses, including ear pinna movements, change in head position and, at higher intensities, startle. Another broadly used approach is recording of cardiovascular variables - heart rate and arterial pressure; in fact it is the direction of cardiac changes that is currently believed to differentiate the orienting response (bradycardia) from the defense response (tachycardia) in rodents. This framework however represents substantial difficulties in interpreting our previous (Kabir et al., 2010) and current data: indeed, if lack of 
bradycardia signifies that the orienting response is not engaged, then how is our isolated respiratory response to be classified? This brings us to the suggestions that: (i) the phenomena that we have described and named so far as "respiratory arousal" is in fact a respiratory component of the orienting response; and (ii) that this respiratory component is more sensitive (i.e., triggered by stimuli of lower intensities) than HR, cutaneous vasoconstrictor, and motor response components.

ADVANTAGES OF RESPIRATORY MEASURES AS AN INDEX OF AROUSAL It is also obvious from our study that compared to cardiovascular measures, respiratory arousal responses have much better temporal resolution. The reason for this is in that while cardiovascular and respiratory responses may originate in the same alarm-related higher brain areas, the descending pathways mediating them are totally different. Sympathetic pathways that trigger tachycardia and cutaneous vasoconstriction are relatively slow in terms of both axonal propagation and post-synaptic effects. In addition, as cutaneous vasomotion is actively involved in thermoregulation in rats (Blessing, 2003; Ootsuka et al., 2008), it is possible that stimulus-induced cutaneous vasoconstriction may sometimes interfere with thermoregulatory vasodilation, resulting in relatively smaller or no responses to mild stimuli when heat dissipation is the priority. In contrast, respiratory responses are rapid as they are executed by striatal muscles innervated by rapidly conducting axons of spinal motoneurons. Importantly, the timing of respiratory responses is in the same range as timing of EEG evoked response potentials (100-300 ms), but in contrast to the latter, they can be more-readily assessed on a single-trial basis. We thus believe that these rapid respiratory arousal responses in rat represent a new and very useful psychophysiological index of behavior. Indeed, in contrast to other autonomic measure, they represent a "real-time" window into sensory processing. Of note, in

\section{REFERENCES}

Barry, R. J. (1977a). Failure to find evidence of the unitary OR concept with indifferent low-intensity auditory stimuli. Physiol. Psychol. 5, 89-96.

Barry, R. J. (1977b). The effect of "significance" upon indices of Sokolov's orienting response: a new conceptualisation to replace the OR. Physiol. Psychol. 5, 209-214.

Barry, R. J. (1978). Physiological changes in a reaction-time task: further problems with Sokolov's dimension of stimulus "significance”. Physiol. Psychol. 6, 438-444.

Barry, R. J. (1979). A factor-analytic examination of the unitary OR concept. Biol. Psychol. 8, 161-178.

Barry, R. J. (2006). Promise versus reality in relation to the unitary orienting reflex: a case study examining the role of

addition to the above mentioned advantages, respiratory measures are simple and non-invasive.

\section{RELEVANCE TO PRELIMINARY PROCESSING THEORY}

Current mechanistic understanding of the "central command" circuitry responsible for the OR is still quite rudimentary, and our data provide a substantial advance in this field. The term refers to the brain area(s) that initiate and coordinate integrative autonomic responses to external stimuli. Sokolov's unitary OR model implies that once activated, the central command initiates simultaneous changes in multiple physiological variables. Our finding do not support this model but rather are in accord with Barry's view (Barry, 2009) that different attributes/dimensions of sensory stimuli are differentially reflected in the pattern of the response components. More specifically, the existence of isolated respiratory responses suggests that mid- or forebrain central command neurons possess a certain level of specialization already at quite a high level of the neuraxis. One of the candidate brain region containing "command neurons" is the $\mathrm{DMH}$, and it is noteworthy that functional segregation of neurons provoking cardiovascular vs. respiratory responses has been described in the DMH (Tanaka and McAllen, 2008).

\section{CONCLUSION}

Tachypneic responses in rat are a new and highly sensitive index of sensory-induced arousal, clearly superior to cardiovascular and motor indices, and comparable to EEG. They likely reflect novelty detection as part of the OR.

\section{ACKNOWLEDGMENTS}

We are grateful to Professor Bill Blessing (Flinders University) for the assistance with experiments measuring EEG and tail blood flow.

part renaissance, part revolution. Pharmacol. Biochem. Behav. 71, 469-480.

Garcia, J. N., Pedersen, N. P., Nalivaiko, E., and Blessing, W. W. (2001). Tail artery blood flow measured by chronically implanted Doppler ultrasonic probes in unrestrained conscious rats. J. Neurosci. Methods 104, 209-213.

Harver, A., and Kotses, H. (1987). Effects of auditory stimulation on respiration. Psychophysiology 24, 26-34.

Hilton, S. M. (1982). The defencearousal system and its relevance for circulatory and respiratory control. J. Exp. Biol. 100, 159-174.

Kabir, M. M., Beig, M. I., Baumert, M., Trombini, M., Mastorci, F., Sgoifo, A., Walker, F. R., Day, T. A., and Nalivaiko, E. (2010). Respiratory pattern in awake rats: effects of motor activity and of alerting stimuli. Physiol. Behav. 101, 22-31.
McNamara, F., Wulbrand, H., and Thach, B. T. (1998). Characteristics of the infant arousal response. J. Appl. Physiol. 85, 2314-2321.

Nalivaiko, E., Beig, M. I., Xavier, C., and Fontes, M. (2010). Dorsomedial hypothalamus and medullary raphe mediate respiratory arousal responses in rats. FASEB J. 24, 1019.16.

Ootsuka, Y., Blessing, W. W., and Nalivaiko, E. (2008). Selective blockade of 5-HT2A receptors attenuates the increased temperature response in brown adipose tissue to restraint stress in rats. Stress 11 , 125-133.

Pavlov, I. P. (1927). Conditioned Reflexes. New York: Dover.

Reyes del Paso, G. A., and Vila, J. (1993). Respiratory influences on the cardiac defense response. Int. J. Psychophysiol. 15, 15-26.

Sgoifo, A., Stilli, D., Medici, D., Gallo, P., Aimi, B., and Musso, E. (1996). 
Electrode positioning for reliable telemetry ECG recordings during social stress in unrestrained rats. Physiol. Behav. 60, 1397-1401.

Sokolov, E. N. (1960). "Neuronal models and the orienting reflex," in The Central Nervous System and Behavior, ed. M. A. Brazier (New York: Macey), 187-276.

Sokolov, E. N. (1963a). Higher nervous functions: the OR. Annu. Rev. Physiol. 25, 545-580.
Sokolov, E. N. (1963b). Perception and the Conditioned Reflex. Oxford: Pergamon.

Tanaka, M., and McAllen, R. M. (2008). Functional topography of the dorsomedial hypothalamus. Am. J. Physiol. 294, R477-R486.

Conflict of Interest Statement: The authors declare that the research was conducted in the absence of any commercial or financial relationships that could be construed as a potential conflict of interest.

Received: 26 August 2011; accepted: 13 December 2011; published online: 03 January 2012.

Citation: Nalivaiko E, Bondarenko E, Lidström A and Barry RJ (2012) Respiratory component of the orienting reflex: a novel sensitive index of sensoryinduced arousal in rats. Front. Physio. 2:114. doi: 10.3389/fphys.2011.00114
This article was submitted to Frontiers in Integrative Physiology, a specialty of Frontiers in Physiology.

Copyright (c) 2012 Nalivaiko, Bondarenko, Lidström and Barry. This is an open-access article distributed under the terms of the Creative Commons Attribution Non Commercial License, which permits non-commercial use, distribution, and reproduction in other forums, provided the original authors and source are credited. 